\title{
Créer, résister, écrire : art, imaginaire et engagement
}

\section{Florent Gaudez*}

\section{Résumé}

Cet article vise à mettre en question le potentiel révolutionnaire de l'art à travers la relation forme/contenu au sein de la dimension esthétique, en supposant que, si la révolution réside dans un autre monde qui est aperçu avant d'être construit, il est important de l'approcher à travers les représentations mentales que l'artiste est capable de faire surgir en les faisant passer du contenu à la forme. Le texte propose notamment d'analyser le thème de la relation entre l'art et la politique, à partir de la posture de Julio Cortázar à l'époque de la révolution cubaine, vers la question des modes d'engagement de l'artiste en tant qu'intellectuel latino-américain. Une posture qui illustre le postulat de Herbert Marcuse, selon lequel le potentiel politique de l'art réside dans sa dimension esthétique.

Mots-clés : Imaginaire, Art, Critique, Création, Écriture, Résistance.

*Université Grenoble Alpes. Grenoble, France 
Create, resist, write: art, imagination and political commitment

\section{Abstract}

This article aims to examine the revolutionary potential of art by looking into the relation form / content within the aesthetic dimension, assuming that, if revolution resides in another world, one that is envisaged before being built, it is important to approach it through mental representations, which the artist is able to engender, turning them from content into form. The text proposes, particularly, to analyze the relationship between art and politics, based on the stance taken by Julio Cortázar at the time of the Cuban revolution, on the matter of the artist's modes of political commitment as a Latin American intellectual. A stance that illustrates the postulate of Herbert Marcuse, that the political potential of art lies in its aesthetic dimension.

Keywords: Imaginary, Art, Critical, Creation. Writing, Resistance.

Créer n'est pas communiquer mais résister (...)

On écrit en fonction d'un peuple à venir qui n'a pas de langage (...)

L'acte de résistance a deux faces : Il est humain et c'est aussi l'acte de l'art (...)

Seul l'acte de résistance résiste à la mort,

soit sous la forme d'une œuvre d'Art, soit sous la forme d'une lutte des hommes.

(Gilles Deleuze, 1987)

Nous sommes une armée de rêveurs,

et pour cette raison nous sommes invincibles.

(Sous-commandant Marcos, 1996)

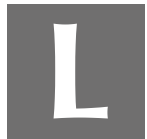

a réflexion que je souhaite proposer ici a pour origine une rencontre : celle de la citation de Gilles Deleuze, en exergue de cet article, entrant en résonnance avec d'autres rencontres faites dans l'espace de la production artistique, sur les plans musical et littéraire en particulier. 
Ainsi, un chanteur français bien connu, Bernard Lavillier, dans son dernier album intitulé Baron samedi, renvoie au vaudou et aux caraïbes en questionnant les rapports entre l'art et le politique, entre la création et la résistance. Par exemple la chanson Tête chargée demande très directement : «Que peut l'art contre la misère noire ? / La musique contre la solitude ? / Les artistes contre les habitudes?».

Ces quelques questions illustrent parfaitement le souci de cet article visant à interroger le potentiel révolutionnaire de l'art à travers le rapport forme/contenu au sein de la dimension esthétique (Marcuse, 1979). En effet, si la révolution repose sur un autre monde aperçu avant d'être construit alors il est important de l'approcher à travers les représentations mentales que l'artiste est capable de faire surgir en les faisant passer du contenu à la forme.

Un autre exemple actuel puisé cette fois-ci dans le monde littéraire est celui de l'écrivaine états-unienne Toni Morrison qui rappelait récemment :

J'ai une vision très différente du rapport entre l'art et la politique que celle de la plupart des gens. Je pense que tout art véritable est politique, et tenter d'en faire quelque chose d'apolitique est en soi un acte politique. Dans ses pièces, Shakespeare parle de gouvernement, de guerre, de pouvoir, et tout cela est politique à mes yeux. Avec la période de l'anticommunisme, on a fait du mot « politique» un mot sale. En réaction à ce qui se passait en URSS, on a décidé aux Etats-Unis que l'art ne devait être qu'esthétique. Alors on s'est mis à dévoyer le sens du mot politique, l'assimilant à la propagande, à quelque chose de sale. Tout mon travail à moi en écrivant est de restaurer le lien entre le politique et la littérature dans le meilleur sens du terme (Morrison, 2013).

On voit bien l'importance qu'elle accorde aux mots, au choix des termes, or, le langage (sous toutes ses formes y compris non verbales) est en effet l'instrument spécifique de la dynamique constitutive du social (Castoriadis, 1975). Il est ce qui permet un investissement imaginaire des « conditions réelles d'existence », investissement imaginaire produisant une reformulation du symbolique (Leenhardt, 1999). 


\section{Une controverse art/politique}

Mais s'il est important de pouvoir s'exprimer, il faut aussi savoir prendre du recul vis-à-vis de certaines évidences. Ainsi Gilles Deleuze (1990, p. 177) nous alertait déjà sur le fait que : «Les forces de répression n'empêchent pas les gens de s'exprimer, elles les forcent à s'exprimer ». $C^{\prime}$ est pourquoi je propose de réfléchir dans un cadre plus général à la question du rapport entre art et politique en m'appuyant entre autres sur la posture de Julio Cortázar issue de la controverse qui l'opposat (1969-70) à l'écrivain argentin Oscar Collazos et à Roberto Fernandez Retamar à l'époque de la révolution cubaine sur la question des modes d'engagement de l'artiste en tant qu'intellectuel latino-américain.

Les jeunes révolutionnaires tel Collazos - fraichement rentré d'un séjour à Cuba - ne jurent plus que par cette nouvelle déesse qu'est la « réalité » dont le nom n'est pas sans évoquer, dans la mémoire de leurs aînés les consternantes aberrations du « réalisme socialiste ». C'est avec une très œdipienne donc meurtrière agressivité que ces nouvelles générations exigent de Cortázar qu'il fasse une littérature « au service du peuple $»^{\mathbf{1}}$. Mais Cortázar reproche sévèrement à ces jeunes contestataires de tenir le peuple en piètre estime quand ils affirment que l'écrivain doit «se pencher »

\footnotetext{
${ }^{1}$ Ainsi que I'indique Karine Berriot, l'occasion leur en est donnée en avril 1970, à la Cité universitaire de Paris, lors d'une manifestation intitulée « Amérique latine non officielle». Au nombre des écrivains conviés, Julio Cortázar et Mario Vargas Llosa vont, en leur qualité de «têtes d'affiche », faire office aussi bien de «têtes de Turcs» tout au long de houleuses réunions. Cette fois encore la polémique fournit à Cortàzar l'occasion d'approfondir certains thèmes de réflexion déjà posés dans la lettre adressée à son ami le poète cubain Roberto Fernández Retamar en mai 1967 (et incluse dans Dernier round) ; c'est ainsi qu'en mai de la même année 1970, il rédige un texte, Viaje alrededor de una mesa (Voyage autour d'une table) qui porte à la connaissance de ses compatriotes d'Amérique latine l'opinion qu'il se fait de lui-même dans la spécificité de sa tâche : «En tant qu'écrivain, je ne m’oppose pas à ce que mon activité soit mise sur le même plan que n'importe quelle autre profession ou labeur humain, et je n'ai pas le moindre sentiment de privilège dans la mesure où ce que j'écris ne me semble ni supérieur ni inférieur à ce que fait le médecin dans son cabinet ou le typographe dans l'imprimerie. Je n'exige qu'une chose, c'est que l'on parle d'une matière en connaissance de cause... » (Berriot, 1988, p. 247).
} 
sur lui, et ainsi abaisser artificiellement le niveau de compréhension de ses textes pour se montrer un bon démocrate. Cette revendication démagogique blesse son sens de la dignité, et lui paraît être même, avec l'esprit de sérieux, l'expression du mal qui guette toujours les révolutions : « Hélas! Hélas les révolutions semblent porter en elles une tendance à la stratification [...] Ces révolutions, dans leur phase initiale, avaient adopté des formes dynamiques, des formes ludiques [...] Mais avec une fréquence assez accablante, après cette première étape, les révolutions s'institutionnalisent, elles commencent à se remplir de chitine, à se transformer en coléoptères » (1986, p. 179-80).

\section{Engagement cortázarien et révolution littéraire et politique}

Ce sur quoi je voudrais focaliser aujourd'hui c'est surtout la posture de Julio Cortázar qui, je le rappelle, est un écrivain argentin (1914-1984) qui a vécu la première moitié de sa vie à Buenos-Aires et la seconde à Paris où il a fini par être naturalisé français sur la fin de sa vie. Il est très connu pour sa littérature parfois qualifiée de «néo-fantastique » en prise permanente avec le quotidien, mais aussi pour ses engagements politiques aux côtés des révolutions latino-américaines en particulier (Cuba, Nicaragua...) ce qui l'a aussi amené, entre autres, à être membre du Tribunal Russel.

L'Argentine était à l'honneur au Salon du Livre de Paris qui s'est tenu du 21 au 24 mars 2014 et à l'occasion duquel on a célébré le centenaire de la naissance de Julio Cortázar qui, par l'écriture, a touché à tous les registres de l'avant-garde contemporaine, cherchant de nouveaux horizons dans le sens de la représentation de la totalité humaine². Angel Rama (1980, p. 8) précise que :

\footnotetext{
${ }^{2}$ Je l'entendrai ici dans le sens de la Socio-anthropologie et du concept de phénomène social total (Marcel Mauss). C'est aussi la question de la diversité de l'humain, dans l'espace et dans le temps, qui est ici en jeu, mais aussi celle de l'invention du social qui contribue à la réalisation de pratiques et de symboliques différenciées à travers l'ensemble des institutions sociales (les « arrangements sociaux fondamentaux», mais aussi les croyances, les modes de conduites, en termes de pratiques et de représentations).
} 
Comme les grands de l'avant-garde européenne et latino-américaine, de Picasso à Neruda, Cortázar postule l'équivalence des deux avant-gardes (l'artistique et la politique) parce qu'elles sont toutes deux des moyens intrépides de reconquérir la plénitude dynamique de l'humanité.

On sent peut-être apparaître là un danger, celui qui, dans les périodes révolutionnaires, impose à l'art des canons, tactiquement afin d'occulter un passé trop proche ou primairement par insuffisance d'imagination esthétique, bâillonnant alors les capacités créatrices et réduisant l'héritage culturel. Mais sur l'engagement de l'écrivain (et de l'artiste en général), Julio Cortázar prend nettement position et ce, de manière très personnelle (1986, p. 173-5) : «J'ai toujours vécu dans un monde de littérature qui est aussi un monde ludique, car c'est pour moi la même chose. II m'était absolument impossible d'accepter un engagement qui aurait été une obédience au devoir exclusif de ne m'occuper que de questions idéologiques ».

Après une première période de sa vie dominée par l'indifférence politique, la biographie de Cortázar prend un tournant décisif avec les événements de Cuba. À la suite d'un voyage sur I'île, I'écrivain revient à Paris avec des préoccupations d'un autre ordre : idéologiques et géopolitiques (« je ne fais pas de la politique » précise-t-il). Il choisit donc d'avoir une démarche « engagée » en prenant maintes précautions afin que sa littérature n'en souffre pas : «II y a des écrivains engagés qui croient qu'il faut faire une littérature politique et les résultats politiques sont médiocres... et les résultats littéraires encore plus médiocres »(Cortázar, 1977, s/i). La mission de l'art et des artistes est bel et bien, pour Cortázar, de poser des questions, de proposer des énigmes ; la science étant là pour tenter de donner des réponses. Face aux rapports entre œuvre, artiste et contexte historique, Cortázar pense qu'il faut avoir une vision marxiste des choses. C'est-à-dire qu'il faut, selon lui, savoir saisir ce qu'est une nouvelle génération; des gens plus jeunes certes, mais surtout qui vivent dans un monde différent avec des problèmes différents, ce qui imprègne nécessairement leur littérature.

Dans un article publié le 9 octobre 1983, dans le journal El País de Madrid, Cortázar explique comment, dans le contexte des mouvements de 
libération de Cuba et du Nicaragua, il fait des critiques pour ces mouvements et non pas contre eux et qu'il s'agit là d'une différence fondamentale avec la critique qui les rejette intégralement même si elle ne le reconnaît pas explicitement. II conclut :

Devant cette perspective, je ne crois qu'au socialisme comme possibilité humaine ; mais ce socialisme doit être un phénix permanent, se dépasser dans un processus constant de renouvellement et d'invention ; et cela ne peut s'obtenir qu'à travers une autocritique dont ces notes ne sont que de vagues petits fragments (Cortázar, 1983).

Effectivement, ainsi que le soulignait Jacques Rancière en se référant à Michel Foucault : «II faut tenir compte du caractère historique de la littérature et la prendre comme formation discursive, épistèmê, dirait Foucault » (Rancière, 1998).

\section{Jeu entre imaginaire et apparences}

La position de l'écrivain est délicate, extérieur à l'épopée militante, il ne cherche qu'à s'attacher, à travers sa démarche littéraire, à ce qu'il nomme «l'exercice imaginaire de compréhension», position révolutionnaire dans l'individualisme et donc suspecte aux yeux des révolutionnaires. Mais son idée est que : «On ne doit pas sacrifier la littérature à la politique ni galvauder la politique sur les autels d'un esthétisme littéraire. Je ne croirais pas au socialisme comme destin historique pour l'Amérique latine s'il n'était pas mû par des raisons d'amour » (Cortázar, 1983). Cortázar se place volontairement en décalage par rapport au réel, selon lui, I'homme consiste en un ensemble de virtualités ne pouvant être captées et mises en œuvre selon les processus rationnels auxquels nous sommes habitués; le monde est une nébuleuse composée de «parcelles dynamiques» où les leviers de la vie et de l'action sont l'imagination et le rêve (Marelle). En exploitant « les failles de l'apparence », l'auteur cherche à donner une forme poétique à la « déformation sournoise que le quotidien codifie» (Fell, 1980, p. 56), s'affirmant ainsi comme « un anarchiste amoureux de l'ordre cosmique, mais jamais de l'ordre nouveau ou du slogan qui fait marquer le pas à cinq ou 
sept cents millions d'hommes en une parodie d'ordre » (ibid.). Le ludique est pour Cortázar la condition sine qua non de l'exercice de la liberté pour I'artiste en général, et il fait allusion en particulier aux « jeux du temps » auxquels se livrait Marcel Duchamp : carambolages réduisant l'avant et I'après à de simples commodités historiques. Le jeu est un dépaysement, accomplissement d'une cérémonie qui se déroule vers l'état final qui la couronne, les contes montrent ce dépaysement, les romans en débattent « dialectiquement» (Fell, 1980). L'auteur est pris à son propre jeu, ce «vaet-vient d'étoiles, de palindromes et d'anagrammes», véritable défi au sens commun. Fondamentalement subversifs, l'humour et le jeu remettent en cause la prétendue « fidélité » du réalisme qui « casse les pieds » à Cortázar : « rien de plus comique que le sérieux envisagé comme valeur préalable à toute littérature importante » Fell, 1980.

Cette posture cortázarienne est par exemple très présente, implicitement ou explicitement, dans les écrits du Sub (Sous-Commandant insurgé Marcos). Cortázar est même souvent cité ès-qualité mais parfois Marcos le convoque sous forme de farces, de clins d'oeils, ou encore sur le mode « à la manière de... ». Ainsi la préface de Marcos à l'édition des communiqués de l'E.Z.L.N. (iYa Basta!) (Sous-Commandant Marcos, 1996, p. 5-15), où, sous couvert d'un communiqué politique extrêmement sérieux, il narre un épisode tout-à-fait quotidien de la vie militaire des insurgés en pleine jungle Lacandone qui s'achève dans des circonstances totalement fantastiques et surréalistes puisque en recherchant l'origine de la panne d'un poste radio de campagne l'on découvre en démontant son panneau arrière qu'il était rempli d'une nuée de perroquets multicolores et d'aras : « Je réussis à compter jusqu'à dix-sept petits perroquets, huit aras femelles et trois mâles, tous se bousculant dans leur sortie » (Ibid., p. 8). Le tout étant finalement signé, comme à l'habitude, du solennel «Depuis les montagnes du Sud-Est mexicain, insurgé Marcos ». Ce n'est qu'ensuite que l'on découvre le Post scriptum : «Gros oubli. J'oubliais que l'objet de la présente est : ... » (Ibid., p. 10). Et il s'engage alors dans l'exposé du véritable contenu de son communiqué politique qui s'étend sur une demi- 
douzaine de pages, c'est-à-dire autant que pour l'histoire quotidienne et fantastique qui constitue l'ensemble du corps du communiqué.

Ce chassé-croisé Cortázar/Marcos, constituant une structure chiasmatique Littérature/Politique vs. Politique/Littérature illustre clairement le postulat d'Herbert Marcuse (1979) selon lequel le potentiel politique de I'art réside dans sa dimension esthétique, son rapport à la praxis étant faible et décevant. Plus une œuvre d'art est immédiatement politique, plus elle perd son pouvoir de décentrement et la radicalité, la transcendance de ses objectifs de changement.

\section{Le passage du contenu à la forme}

Herbert Marcuse met en cause, à travers l'esthétique marxiste, l'orthodoxie dominante comme interprétation de la qualité et de la vérité $\mathrm{d}^{\prime}$ 'une œuvre d'art, y compris littéraire, par rapport aux moyens de production. S'il rejoint l'esthétique dite marxiste pour dire que l'œuvre d'art a une fonction et un potentiel politique dans sa relation avec le contexte et les relations sociales, il s'en écarte néanmoins sur plusieurs points, car c'est dans l'art lui-même (dans la forme esthétique en tant que telle) que Marcuse trouve le potentiel politique, et pour lui, il y a autonomie entre l'art et les rapports sociaux donnés (l'art s'oppose à ces rapports sociaux et les transcende, et subvertit la conscience dominante, c'est-à-dire l'expérience ordinaire). Marcuse traite de l'art en général en s'attachant à la littérature en particulier, pour lui l'art est révolutionnaire dans le sens d'un changement radical de style, de technique, comme annonciateur de changements dans la société, mais surtout par la forme donnée au contenu (il cite Brecht, Kafka, Beckett), le contenu (vérité établie) n'apparaît plus que distancé et médiatisé. II renvoie par ailleurs à Lucien Goldmann (1964, p. 44) pour qui la question essentielle est de se demander « comment se fait la liaison entre les structures économiques et les manifestations littéraires dans une société où cette liaison a lieu en dehors de la conscience collective », et

\footnotetext{
3 « La vérité est la mort de l'intention », (Benjamin, 1991, p. 216).
} 
rappelle que celui-ci a problématisé l'esthétique marxiste dans le contexte du capitalisme avancé où le prolétariat n'est plus la négation de la société mais y est au contraire largement intégré : I'esthétique marxiste doit alors faire face à une réalité particulière où des formes authentiques de création culturelle ne renvoient pas systématiquement à la conscience - même possible - d'un groupe social particulier. Ainsi, l'œuvre d'art n'est pas révolutionnaire parce que s'adressant à la classe ouvrière ou parce que préparant la révolution, mais, comme le rappelle Marcuse, parce qu'elle se renvoie à elle-même en tant que contenu devenu forme.

Alors que T.-W. Adorno (1967, p. 97) ${ }^{4}$ affirmait que les œuvres qui se présentent sans résidu au regard et à la pensée ne sont pas des œuvres d'art, de son côté Brecht (1967, p. 411), pourtant loin d'être un zélateur de l'autonomie de l'art, affirmait que « une œuvre qui ne fait pas preuve de souveraineté vis-à-vis de la réalité et qui n'octroie pas de souveraineté au public [peuple] sur la réalité n'est pas une œuvre d'art », tandis que plus tard en Sorbonne Louis-Vincent Thomas, élargissant la question à l'espace de l'imaginaire, écrivait quant à lui que :

Si l'imaginaire déroute par ses excès, ses pulsions incontrôlées, ses divagations effrénées, il n'empêche qu'il traduit l'homme total que la société techniciste et rationaliste ne cesse d'atrophier. Le système n'a que deux attitudes possibles à l'endroit de l'imaginaire : le récupérer dans le circuit de la rentabilité, ce qui ne manque pas de se produire en maintes circonstances ; le tourner en dérision en le tenant pour errement de poète ou de gauchiste. Il faut en tout cas le contrôler, l'extirper, le maintenir en marge (Thomas, 1979, p. 16-7).

\footnotetext{
${ }^{4}$ Il précise ainsi : «Il existe des œuvres d'art dont la qualité ne fait pas de doute, mais qui, au moins si elles sont jugées selon les critères de leur influence quantitative, sont négligeables au niveau social, et devraient donc, à en croire Silbermann, être exclues du domaine de recherches. Mais une telle exclusion entraînerait un appauvrissement de la sociologie de l'art : les œuvres d'art les plus valables lui échapperaient. Le fait que celles-ci ne parviennent pas à exercer une influence sociale considérable est aussi bien un fait social que le contraire. La sociologie de l'art doit-elle abdiquer devant ce problème ?».
} 


\section{L'Image-Temps}

Le projet révolutionnaire ne considère l'idée d'un homme aliéné que pour le libérer et le transfigurer en homme total disposant de lui-même ; ainsi son prédicat et son objet sont-ils confondus et le changement dont il est porteur se situe-t-il au niveau de la totalité et de l'universalité. La totalité du projet révolutionnaire serait-elle de l'ordre de l'image, plus que de celui du rêve ; de la réalité, plus que de l'utopie ? Selon André Decouflé cette totalité fonde le projet en réalité nécessaire dans la banalité la plus immédiate de son quotidien; le projet révolutionnaire implique que la révolution se suffise à elle-même, et exclut la destruction de l'homme puisqu'il est sa régénérescence :

Changer la vie et le monde, implique création collective quotidienne d'un nouvel « ensemble historique » situé dans la durée, qui joint l'histoire de jadis à I'histoire d'aujourd'hui - le quotidien - et au monde de demain : le projet révolutionnaire est immanent au monde et dans le même temps prise de possession naïve du quotidien de la vie. Quotidienneté de la révolution en actes et immanence du projet révolutionnaire ne s'opposent pas : elles sont les deux faces d'une même réalité (Decouflé, 1968, p. 41).

Je situe ces deux versants chacun dans l'une des deux dimensions temporels mises en évidence par Cornélius Castoriadis : la quotidienneté de la révolution en actes dans le temps identitaire du faire social, et l'immanence du projet révolutionnaire dans le temps imaginaire du représenter social. Il serait ainsi question de prendre distance avec le temps « du faire social », le temps « identitaire » pour reprendre les termes utilisés par Castoriadis (1975) dans sa distinction entre temps « identitaire » et temps « imaginaire ». Le temps identitaire est le temps où le « faire social » est rendu possible, le temps imaginaire est le temps permettant le «représenter social»; Castoriadis prolonge ici la pensée de Freud ('inconscient ignore le temps et ignore la contradiction), en affirmant que l'inconscient constitue un « lieu » où le temps « identitaire » n'existe pas, que l'inconscient n'est que comme flux indissociablement représentatif/affectif/intentionnel. Or le 
sociologue ne peut se passer d'hypothèse sur les déterminants de l'action sociale, la misère n'explique pas la révolte, encore moins la révolution, car celle-ci suppose un but, une image de la liberté qui permette de prendre conscience de la misère. C'est cette image qui est à la base de l'élaboration du projet. Et si la révolution repose sur un projet en vue d'un autre monde aperçu avant d'être construit, alors il est particulièrement intéressant de l'approcher d'abord par ses représentations mentales.

Ernesto « Che » Guevara disait que le révolutionnaire ne cherche pas le bonheur, mais une vie autre, qui puisse donner à son projet une dimension autre que celle de la béatitude du quotidien ; effectivement, ce qui fait le révolutionnaire c'est qu'il ne choisit pas ce vers quoi on tend en général : la paix, considérant que si l'on choisit la paix, on n'est pas sûr d'avoir la liberté, il faut accepter de ne pas avoir la paix si l'on veut la liberté. Idée que le « Che » résume à sa façon : «Plutôt guérillero que ministre! », et Castro (1962) à la sienne : « le devoir d'un révolutionnaire est de faire la révolution $»$.

Au plan littéraire, Cortázar reprend cette idée pour expliquer que : «Le roman révolutionnaire, n'est pas seulement celui qui dit la révolution, mais c'est surtout celui qui révolutionne le roman [...] Nous avons plus que jamais besoin de Che Guevara du langage, de révolutionnaires de la littérature plutôt que de lettrés de la révolution» (1980b, p. 63-81). L'intellectuel surréaliste n'est pas moins révolutionnaire que le guérillero, il ne l'est que de façon différente. Dans le même esprit, l'un des personnages de Julio Cortázar déclare : "II n'est pas sûr qu'entre Lénine et Rimbaud il y ait une telle différence » (Livre de Manuel).

\section{Dimension esthétique et révolution}

Chez Marcuse la catharsis possède un caractère rédempteur, une fonction de réconciliation entre la réalité établie et les « forces rebelles » qui transcendent la détermination de classe : «La catharsis elle-même se fonde sur le pouvoir de la forme esthétique d'appeler le destin par son nom, 
de démystifier cette force, de donner la parole aux victimes » (Marcuse, 1979 , p. 24). Elle relève plus de I'ontologique que du psychologique et en se dressant contre la réalité établie, elle se fonde sur les propriétés de la forme elle-même : "s son ordre non répressif, sa capacité cognitive et l'image qu'elle offre de la fin de la souffrance » (Ibid., p. 70).

Mais à travers l'art, la subversion de l'expérience et la rébellion qu'elle engendre contre le principe de réalité ne peuvent pas, d'après Marcuse, se cristalliser dans une praxis politique et c'est précisément sur cette nonidentité que se fonde le potentiel radical de l'art ; d'où la question qu'il nous pose : «Comment ce potentiel peut-il trouver une représentation valide dans une œuvre d'art et comment peut-il devenir un facteur de transformation de la conscience ?» (lbid., p. 51) à la suite de ce qu'il avait formulé quelques dizaines de pages auparavant :

En ce sens, l'art est «pour l'art » dans la mesure où la forme esthétique révèle des dimensions de la réalité qui sont réprimées ou font l'objet de tabous. La poésie de Mallarmé en est un exemple extrême : elle suscite des modes de perception, d'imagination, de gestes, une fête sensuelle qui réduit en miettes l'expérience quotidienne et annonce un principe de réalité différent (Ibid., p. 33).

On se souvient de Julio Cortázar $(1980 b)^{5}$ déclarant : « ... les matérialistes te disent qu'il faut écrire sur la réalité de tous les jours, et sur la destinée des peuples. [...] on continue à nous casser les pieds avec la littérature à thèse et le réalisme » et par ailleurs : « La littérature doit faire mouche de partout et non pas seulement du point de vue socio-politique sous le prétexte qu'on appartient au Tiers monde ». Or, ainsi que je l'ai précisé plus haut, pour Marcuse l'œuvre d'art n'est pas révolutionnaire parce que s'adressant à la classe ouvrière ou parce que préparant la révolution, mais, parce qu'elle se renvoie à elle-même en tant que contenu devenu forme. Pour lui, le potentiel politique de l'art réside donc dans sa dimension

${ }^{5}$ Signalons aussi, pour la petite histoire que Cortázar avait un chat surnommé « Théodore W. Adorno » sur lequel il a écrit un très joli texte intitulé «L'entrée en religion de Théodore W. Adorno », in Le tour du jour en quatre-vingts mondes (Cortázar, 1980a. p. 233-6). 
esthétique car, par ailleurs, son rapport à la praxis est faible et décevant ; plus une œuvre d'art est immédiatement politique, plus elle perd son pouvoir de subversion (de décentrement et de radicalité). Ces positions nous ramènent à Cortázar précisant au sujet de sa pratique d'écrivain : «Mais je ne crois pas aujourd'hui comme je l'ai cru commodément autrefois que la littérature de pure création imaginaire me suffise pour sentir que j'ai fait mon devoir d'écrivain [...] je sais aujourd'hui que j'écris pour ; qu'il y a une intention qui s'adresse à un lecteur espéré, dans lequel résiderait déjà la semence de l'homme futur » (Cortázar, 1984, p. 24-5). La distinction sur le plan révolutionnaire entre Breton ou Malraux, Lénine ou Rimbaud, Guevara, Cortázar ou Marcos, ne semble pas, aussi profonde qu'à l'évidence ; idem quant à la relativité des limites action/imagination et engagement/ représentation ${ }^{6}$.

\section{Conclusion : art et émancipation}

Il faut se créer des contraintes pour pouvoir inventer en toute liberté.

Umberto Eco (1985, p. 30)

En s'intéressant à la question de l'art comme fait social, Jean Duvignaud $(1967, \text { p.5 })^{7}$ met en garde contre la simplification qui consisterait à vouloir restituer l'expérience individuelle de la création en se fondant uniquement sur des éléments provenant de l'approche du collectif social stricto sensu. Selon lui il faut saisir la «dynamique de la vie sociale», en intégrant l'expérience de la création comme expérience vivante, en actes, et « comprendre la totalité de l'expérience artistique dans la totalité de I'expérience sociale» (Ibid., p. 34). II me semble important de rapprocher cette position de celle de Roger Bastide écrivant :

\footnotetext{
${ }^{6}$ Limites qu'il est aisé de rapprocher des suivantes : projet révolutionnaire/révolution en actes, fantastique/quotidienneté, imaginaire/réel.

7 Une sociologie de l'art doit chercher à « retrouver les formes de l'enracinement de I'imaginaire dans notre existence collective, sans dogmatisme et sans pédanterie ».
} 
L'art continue le dynamisme social par d'autres moyens. En un mot, si au lieu de considérer le social comme une réalité statique, on le considère comme une réalité dynamique, le producteur d'art est celui, qui par la puissance de son imagination, épouse le mouvement en train de se faire pour le parachever et lui faire signifier son originalité créatrice. L'artiste est moins le reflet de la société que celui qui l'accouche de toutes ses nouveautés (Bastide, 1977, p. 93).

Ce qui est intéressant, en posant les choses de cette manière, c'est que cela confère à l'idée de création une dimension supplémentaire à celle de simple processus de production d'une marchandise. Cette dimension spécifique dans le champ symbolique est celle de la fonction critique de la création artistique. Bien sûr, le créateur et son œuvre sont inscrits en tant que tels dans la réalité sociale, mais ce qui les spécifie dans l'acte de création c'est cette possibilité de montrer quelque chose de nouveau qui peut être n'aurait pas surgi, ou serait advenu d'une autre manière, sans la démarche de l'artiste et ce qui en est issu l'objet d'art ou le texte littéraire en tant qu'œuvre. C'est pourquoi pour Marcuse, la question de la forme est centrale, « C'est dans l'art lui-même, dans la forme esthétique en tant que telle, que je trouve le potentiel politique de l'art » $\left(1979\right.$, p. 9) ${ }^{\mathbf{8}}$, ajoutant :

L'art affirme sa réserve vis-à-vis de la thèse selon laquelle l'heure est venue de changer le monde. L'art témoigne de la nécessité de la libération mais il témoigne aussi de ses limites. Ce qui a eu lieu ne peut plus être défait, ce qui est passé ne peut plus être sauvé ; I'histoire est faute, mais sans rédemption. Eros et Thanatos ne sont pas seulement adversaires mais aussi amants (Marcuse, 1979, p. 79).

Si nous ne pensions pas l'impossible, le possible ne serait pas réalisable. Le monde de l'art est selon Marcuse, Marcos et Cortázar le monde de l'altérité, celui d'un principe de réalité différent qui remplit une fonction cognitive : dire ce qui est indicible autrement et... contredire.

Dans un contexte international à la fois fragile, agité et violent, dans un monde aujourd'hui incertain, instable et globalisé, j'ai envie de ponctuer

${ }^{8}$ Plus de dix ans auparavant, il écrivait : "L'imagination a été touchée par le processus de réification. Nous sommes possédés par nos images, nous souffrons par nos images » (Marcuse, 1968, p. 274). 
ce texte en laissant le dernier mot à un socio-anthropologue, que j'ai déjà mobilisé plus haut, Jean Duvignaud :

\begin{abstract}
Laissons au cimetière les traditions mortes. Seules les traditions vivantes disposent du dynamisme créateur capable d'anticiper sur l'avenir et d'établir entre ceux qui, dans tous les domaines, cherchent à inventer l'avenir des rapports, des correspondances. Il existe une internationale des créateurs. [...] Après tout, le monde humain est fait de plus d'imprévisible que d'inéluctable, et le dynamisme des cultures peut aider à dissoudre les brouillards d'une résistible apocalypse (1984, s/i).
\end{abstract}

C'était il y a plus de trente ans...

Florent Gaudez est socio-anthropologue, professeur de sociologie à L'UFR Sciences de I'homme et de la société de l'Université Grenoble Alpes, chargé de conférences à l'EHESSParis et Vice-Président de I'AFS (Association Française de Sociologie).

$\equiv$ florent.gaudez@univ-grenoble-alpes.fr

\title{
Références
}

1. ADORNO, Theodor W. Thesen zur Kunstsoziologie. In : ADORNO, Theodor W. Ohne Leitbild. Parva Aesthetica. Frankfurt : Suhrkamp, 1967.

2. BASTIDE, Roger. Art et société. Paris : Payot, 1977.

3. BENJAMIN, Walter. Ursprung des Deutschen Trauerspiels. In : BENJAMIN, Walter. Gesammelte Schriften 1. Frankfurt : Suhrkamp, 1991. p. 216.

4. BERRIOT, Karine. Julio Cortàzar. L'enchanteur. Paris : Presses de la Renaissance, 1988.

5. BRECHT, Bertolt. Gesammelte Werke, Vol. XIX. Frankfurt : Suhrkamp, 1967.

6. CASTORIADIS, Cornélius. L'institution imaginaire de la société. Paris : Seuil, 1975.

7. CASTRO, Fidel. Deuxième déclaration de La Havane, 1962. Disponible sur : http://www.fidelcastro.cu/fr/discursos/discours-de-fidel-castro-ruz-la-deuxiemeassemblee-generale-nationale-du-peuple-de-cuba

8. CORTÁZAR, Julio. « Le fantastique du quotidien et le jazz », interview. Jazzhot, v. 43, n. 337, mai 1977.

9. CORTÁZAR, Julio. Le tour du jour en quatre-vingts mondes. Paris : Gallimard, 1980a. 
10. CORTÁZAR, Julio. Paris, « Littérature et révolution ». L'ARC, n. 80, 1980b. p. 63-81.

11. CORTÁZAR, Julio. El destino del hombre era... '1984'. El País, Madrid, 9 oct. 1983. Disponible sur : <https ://elpais.com/diario/1983/10/09/ internacional/434502007_850215.html>.

12. CORTÁZAR, Julio. Entretiens avec Omar Prego. Paris : Gallimard, 1984.

13. DECOUFLÉ, André. Sociologie des révolutions. Paris : P.U.F., 1968.

14. DELEUZE, Gilles. "Qu'est-ce que l'acte de création ? ». Conférence à la FEMIS, 17 mar. 1987.

15. DELEUZE, Gilles. Pourparlers. Paris : Minuit, 1990.

16. DUVIGNAUD, Jean. "Le théâtre et la dérision ». Internationale de I'Imaginaire, n. 0, jan./fév. 1984.

17. DUVIGNAUD, Jean. Sociologie de I'art. Paris : P.U.F., 1967.

18. ECO, Umberto. Apostille au nom de la rose. Paris : Grasset, 1985.

19. FELL, Claude. « Des jeux pour vivre et pour rêver ». L'ARC, n. 80, p. 56-62, 1980.

20. GOLDMANN, Lucien. Pour une sociologie du roman. Paris : Gallimard, 1964.

21. LEENHARDT, Jacques. "Une sociologie des œuvres d'art est-elle nécessaire et possible ?". In: MOULIN, Raymonde (dir.) Sociologie de l'Art. Paris : L'Harmattan, 1999, pp. 385-395.

22. MARCOS, Sous-commandant. iYa Basta ! Les insurgés zapatistes racontent un an de révolte au Chiapas. Tome 1. Paris : Dagorno, 1996.

23. MARCUSE, Herbert. L'homme unidimensionnel. Essai sur I'idéologie de la société industrielle avancée (1964). Paris : Minuit, 1968.

24. MARCUSE, Herbert. La dimension esthétique. Paris : Seuil, 1979.

25. MORRISON, Toni. «Tout art véritable est politique ». Magazine Littéraire, $\mathrm{n}$. 527, p. 85-9, jan. 2013.

26. RAMA, Angel. « Julio Cortàzar, inventeur du futur ». L'ARC, n. 80, p. 8-16, 1980.

27. RANCIÈRE, Jacques. À part Rancière. « Casser I'opposition des mots et des choses $\gg$. Entretien à Robert Maggiori. Libération, 5 mars 1998. Disponible sur : http://next.liberation.fr/livres/1998/03/05/casser-I-opposition-des-mots-et-deschoses_232210

28. THOMAS, Louis-Vincent. Civilisation et divagations. Paris : Payot, 1979. 